\title{
Potentialités de la microfiltration tangentielle sur membranes minérales pour la clarification du jus de pomme de cajou
}

\author{
Fernando Abreu ${ }^{a}$, Ana Mercedes Perez ${ }^{b}$, Manuel DornieR ${ }^{c, d_{*}}$, Max ReYNes ${ }^{c}$
}

\author{
a EMBRAPA, Agroindústria \\ Tropical, Setor de Tecnologia \\ de Alimentos, Av. Dra. Sara \\ Mesquita, 2270 Planalto Pic, \\ 60.511-110 Fortaleza, Ceará, \\ Brasil \\ ${ }^{b}$ Centro Nacional de Ciencia y \\ Tecnología de Alimentos \\ (CITA), Universidad de Costa \\ Rica, Código Postal 2060, \\ San José, Costa Rica \\ ${ }^{c}$ Centre de Coopération \\ Internationale en Recherche \\ Agricole pour le \\ Développement (Cirad), \\ Département Flhor, avenue \\ Agropolis, TA 50/PS4, 34398 \\ Montpellier Cedex 5, France \\ dornier@cirad.fr
}

d École Nationale Supérieure des Industries Alimentaires (Ensia), Section des Industries Alimentaires Régions Chaudes (Siarc), 1101 avenue Agropolis, C.S. 24501, 34093 Montpellier Cedex 5, France

* Correspondance et tirés à part

Reçu le 4 juin 2004

Accepté le 12 octobre 2004

Fruits, 2005 , vol. 60 , p. $33-40$ (C) 2005 Cirad/EDP Sciences All rights reserved

DOI: 10.1051/fruits:2005010

RESUMEN ESPAÑOL, p. 40

\section{Application of cross-flow microfiltration in the production of clarified cashew juice by means of mineral membranes.}

Abstract - Introduction. Cashew apple is often regarded as a by-product of the cashew nut industry, and remains under-developed. Among the various possible technological options for the clarification of the pulpy fruit juices, cross-flow microfiltration on mineral membranes is a particularly promising process. Thus, the objective of our work was to evaluate the advantages of this process for the production of clarified cashew apple juice. Materials and methods. Filtration assays were conducted at $30^{\circ} \mathrm{C}$ in a laboratory-scale pilot equipment with several tubular aluminium membranes. Four membranes were tested, differentiated by the average diameter of their pores $[(0.1 ; 0.2 ; 0.8$ and 1.4) $\mu \mathrm{ml}$. The performances (permeate flux) and the composition (condensed tannins, acidity, ascorbic acid, $\mathrm{pH}$, turbidity, color and soluble dry extract) of the clarified juice obtained were compared for each one of these membranes. The use of an enzymatic liquefaction pre-treatment added to the microfiltration was evaluated. Results and discussion. The permeate flux obtained ranged from (36 to 79$) \mathrm{L} \cdot \mathrm{h}^{-1} \cdot \mathrm{m}^{-2}$. The best results for the study conditions were obtained with the membranes with an average pore diameter of $(0.1$ and 0.2$) \mu \mathrm{m}$. Microfiltration coupled with an enzymatic liquefaction pre-treatment improved the filtration yield by (30 to 60)\%. All the membranes used made it possible to obtain a completely clarified juice in which the ascorbic acid content was very close to that of the fresh juice. On the other hand, the phenolic compounds present in great quantity in the raw apple cashew juice were almost completely eliminated during the process, thus making it possible to considerably decrease the juice astringency. Conclusion. Our work checked the potential of the cross-flow microfiltration process for the production of clarified cashew apple juice. Nevertheless, before considering a future industrial application, the treatment conditions will have to be optimized and the nutritional and sensory qualities of clarified cashew apple juice will have to be better characterized.

Brazil / Anacardium occidentale / cashew apple / fruit juices / clarifying / microfiltration / membranes / polyphenols

\section{Potentialités de la microfiltration tangentielle sur membranes minérales pour} la clarification du jus de pomme de cajou.

Résumé - Introduction. La pomme de cajou, souvent considérée comme un sous-produit de l'industrie de la noix de cajou, reste très peu valorisée. Parmi les diverses options technologiques possibles pour la clarification des jus de fruits pulpeux, la microfiltration tangentielle sur membrane minérale est un procédé particulièrement prometteur. L'objectif de notre travail a donc été d'évaluer l'intérêt de ce procédé pour la production de jus de pomme de cajou clarifié. Matériel et méthodes. Des essais de filtration ont été réalisés à $30^{\circ} \mathrm{C}$ sur une installation pilote de laboratoire munie de diverses membranes tubulaires en alumine. Quatre membranes ont été testées, différenciées par le diamètre moyen de leurs pores $[(0,1 ; 0,2 ; 0,8$ et 1,4$) \mu \mathrm{m}]$. Les performances (densité de flux de perméat) et la composition du jus clarifié obtenu (tannins condensés, acidité titrable, acide ascorbique, $\mathrm{pH}$, turbidité, couleur et extrait sec soluble) ont été comparées pour chacune de ces membranes. L'utilisation d'un prétraitement enzymatique de liquéfaction couplé à la microfiltration a également été abordée. Résultats et discussion. Les densités de flux de perméat obtenues ont été comprises entre (36 et 79) $\mathrm{L} \cdot \mathrm{h}^{-1} \cdot \mathrm{m}^{-2}$. Dans les conditions testées, les membranes de diamètre de pores de $(0,1$ et 0,2$) \mu \mathrm{m}$ ont conduit aux meilleurs résultats. Le couplage de la microfiltration avec un prétraitement enzymatique de liquéfaction a amélioré les performances de la filtration de (30 à 60) \%. Toutes les membranes ont permis d'obtenir un jus parfaitement limpide dont la teneur en acide ascorbique a été très voisine de celle du jus frais. En revanche, les composés phénoliques présents en grande quantité dans le jus de pomme de cajou brut ont été presque totalement éliminés au cours de l'opération permettant ainsi de diminuer considérablement l'astringeance du jus. Conclusion. Nos travaux ont vérifié le potentiel du procédé de microfiltration tangentielle sur la production de jus clarifié de pomme de cajou. Néanmoins, avant d'envisager une future application industrielle, les conditions de traitement devront être optimisées et les qualités nutritionnelle et sensorielle du jus clarifié devront être mieux caractérisées.

Brésil / Anacardium occidentale / pomme cajou / jus de fruits / clarification / microfiltration / membrane / polyphénol 


\section{Introduction}

L'anacardier (Anacardiun occidentale L.) est une plante d'origine brésilienne. Dans ce pays, les cultures d'anacardiers sont passées de (82 000 à 630000$)$ ha entre 1974 et 1994. Cette augmentation spectaculaire est principalement liée au développement, notamment par l'Embrapa, de nouvelles variétés naines précoces. Associées à des techniques culturales adaptées, ces variétés permettent d'obtenir une productivité très élevée pouvant atteindre jusqu'à $5 \mathrm{t} \cdot \mathrm{ha}^{-1}$, à comparer avec les $280 \mathrm{~kg} \cdot \mathrm{ha}^{-1}$ obtenus en cultures traditionnelles. D'après la FAO [1], le Brésil est le troisième producteur mondial de noix de cajou avec une production d'environ $167000 \mathrm{t} \cdot \mathrm{an}^{-1}$ après l'Inde (440 $000 \mathrm{t} \cdot \mathrm{an}^{-1}$ ) et le Nigeria (176 $000 \mathrm{t} \cdot \mathrm{an}^{-1}$ ). Néanmoins, des estimations récentes indiquent qu'aujourd'hui près de 700000 ha d'anacardiers sont exploités au Brésil et que la production totale de noix de cajou avoisine $280000 \mathrm{t} \cdot \mathrm{an}^{-1}$. Environ $99 \%$ de la production nationale est assurée par les États du Ceará, de Piauí et de Rio Grande do Norte, tous localisés dans le nord-est du pays [2]. La filière cajou emploie environ 300000 personnes au Brésil, ce qui lui confère une importance socio-économique considérable.

La pomme de cajou est le faux fruit de l'anacardier. Il correspond au pédoncule hypertrophié de la noix et représente cinq à neuf fois la masse de la noix [3]. La pomme de cajou est le plus souvent considérée comme un sous-produit de l'industrie de la noix de cajou et reste très peu valorisée. Seule une très faible partie des pommes produites est commercialisée sur les marchés locaux, essentiellement en frais [4]. Ainsi, plus de 1 Mt de pommes de cajou seraient perdues par an au Brésil [5]. La pomme de cajou peut pourtant être facilement transformée en jus de fruit. D'une part, les rendements d'extraction sont élevés (> $70 \%$ ), d'autre part, avec un extrait sec soluble compris entre (10 et 12) \%, le jus de pommes de cajou est riche en vitamine $C$ [(2 à 3) $\left.\mathrm{g} \cdot \mathrm{L}^{-1}\right]$. Les acides anacardiques isolés du jus de pomme de cajou semblent présenter des propriétés cytotoxiques contre des cellules tumorales [6]. Ces acides anacardiques associés à l'(E)-2-hexenal, un des 90 composés d'arôme détectés dans la pomme de cajou, auraient une activité antimicrobienne, notamment contre la bactérie Helicobacter pylori $[7,8]$. Compte tenu de ces caractéristiques et de la grande disponibilité de la matière première, la transformation de la pomme de cajou en jus de fruit semble donc intéressante à développer. Cependant, le principal facteur qui limite le développement de ce produit, en particulier sur les marchés internationaux, est son astringence élevée [9]. Cette astringence est liée à la richesse de la matière première en composés phénoliques condensés.

Les jus de fruits tropicaux clarifiés intéressent de plus en plus l'industrie des boissons. En effet, ces jus, le plus souvent utilisés comme ingrédients dans diverses formulations, permettent d'élaborer de nouveaux produits originaux [10]. Dans le cas de la pomme de cajou, le jus clarifié présente un intérêt supplémentaire car il peut être utilisé pour enrichir le produit fini en vitamine $\mathrm{C}$ naturelle à un coût relativement faible. Parmi les diverses options technologiques qu'il est possible d'envisager pour la clarification des jus de fruits pulpeux, la microfiltration tangentielle sur membrane minérale est un procédé particulièrement prometteur. En cours de développement pour le traitement de divers jus de fruits, il permet de produire de façon continue des jus clarifiés sans utiliser d'adjuvants de filtration et présente des performances intéressantes pour une utilisation industrielle [10-12].

Dans ce contexte, l'objectif du travail présenté a été d'évaluer l'intérêt de la microfiltration tangentielle sur membranes céramiques pour la production de jus de pomme de cajou clarifié. Les performances du procédé et son impact sur la qualité du jus de fruit ont été comparés en utilisant quatre membranes de diamètre de pores différent.

\section{Matériel et méthodes}

\subsection{Matière première}

Les pommes de cajou utilisées provenaient d'anacardiers nains, précoces, de la variété CCP-076 (clone cajueiro pacajus) développées par l'Embrapa. Les fruits avaient été 


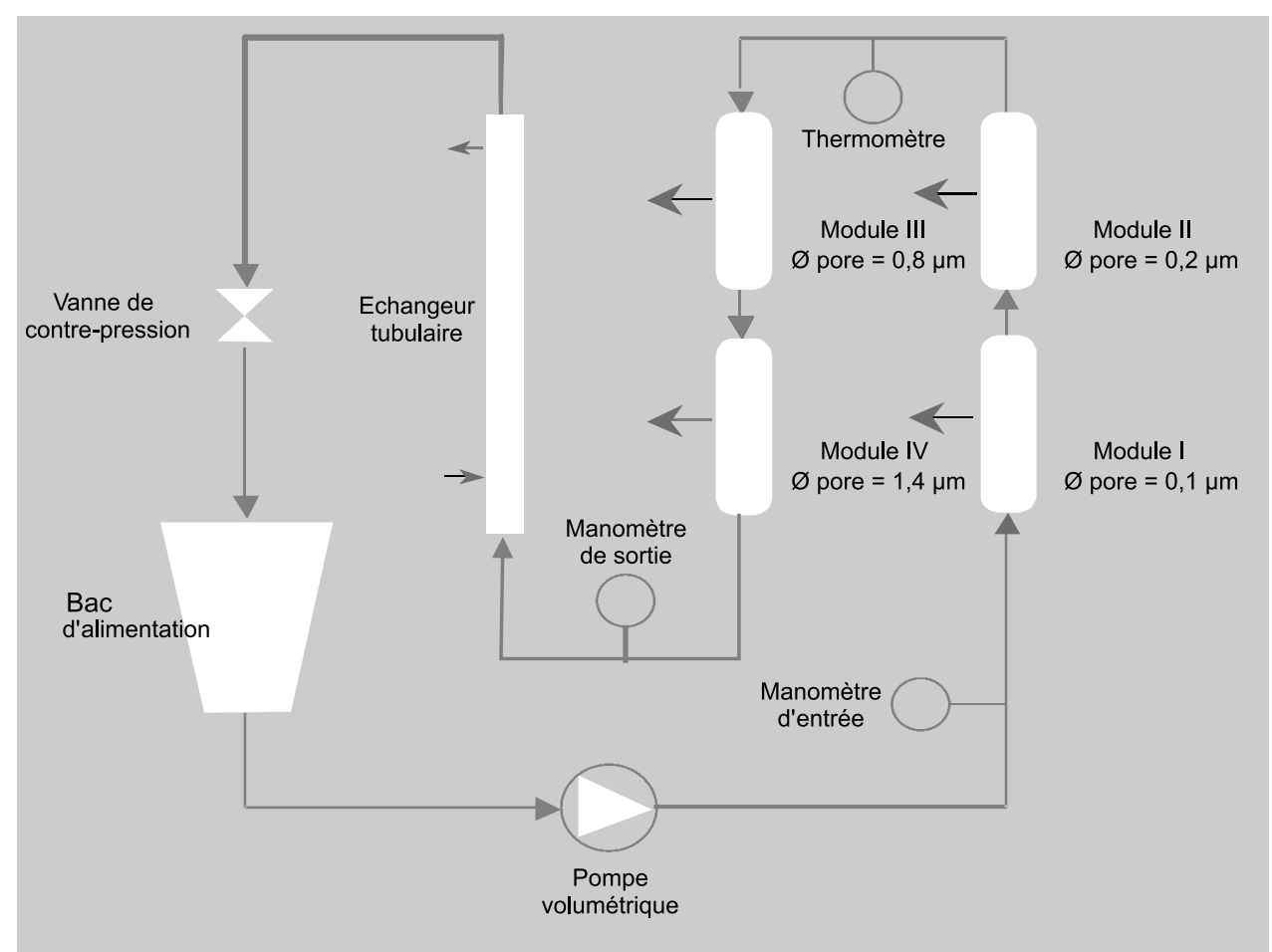

récoltés mûrs dans les plantations de la station expérimentale Agroindustria Tropical de l'Embrapa à Paraipaba, à 120 km de Fortaleza (Ceará, Brésil). Après lavage à l'eau froide, l'extraction du jus a été réalisée par pressage à l'aide d'une presse " expeller" (Ceil, capacité de $300 \mathrm{~kg} \cdot \mathrm{h}^{-1}, 25-28^{\circ} \mathrm{C}$ ). Les rendements d'extraction obtenus ont été de (75 à 80) \%. Le jus a alors été filtré sur un tamis à mailles de $3 \mathrm{~mm}$ pour en éliminer les fibres avant d'être conditionné dans des sachets de $10 \mathrm{~kg}$ et congelé à $-18^{\circ} \mathrm{C}$ jusqu'à son traitement par microfiltration.

\subsection{Microfiltration tangentielle}

La microfiltration du jus de cajou a été réalisée à l'aide d'un pilote de laboratoire (TIA, Bollène, France). D'une capacité de $3 \mathrm{~L}$, l'installation est équipée d'une pompe volumétrique assurant à la fois la mise sous pression du circuit rétentat et la circulation tangentielle (figure 1). Les membranes utilisées sont tubulaires, constituées d'alumine et présentent une surface efficace de $55 \mathrm{~cm}^{2}$ chacune. Quatre membranes céramiques (Pall-
Exekia, Bazet, France) ont été choisies pour les essais. Montées en série dans l'installation (modules I à IV), elles présentent des diamètres de pores de 0,$1 ; 0,2 ; 0,8$ et $1,4 \mu \mathrm{m}$. Les pressions d'entrée $\left(\mathrm{P}_{\mathrm{e}}\right)$ et de sortie $\left(\mathrm{P}_{\mathrm{s}}\right)$ du circuit ont été fixées respectivement à 2,6 bar et 0,5 bar. Pour chacun des modules, la pression transmembranaire moyenne $\left(\mathrm{P}_{\mathrm{tm}}\right)$ a été évaluée à partir de $\mathrm{P}_{\mathrm{e}}$ et $\mathrm{P}_{\mathrm{s}}$ en supposant que le profil de pression était linéaire côté rétentat et en négligeant les pertes de charges générées par les deux coudes présents sur le circuit. La vitesse tangentielle a été comprise entre (6 et 7) $\mathrm{m} \cdot \mathrm{s}^{-1}$. La température a été contrôlée à $(30 \pm 2)^{\circ} \mathrm{C}$, à l'aide de l'échangeur de chaleur tubulaire alimenté par de l'eau froide. Le nettoyage des membranes a été effectué selon un protocole classique [base / acide] adapté à ce type d'installation: $\left[\left(\mathrm{NaOH} 2 \%, 80^{\circ} \mathrm{C}\right.\right.$, $\left.40 \mathrm{~min}) /\left(\mathrm{HNO}_{3} 1 \%, 30^{\circ} \mathrm{C}, 40 \mathrm{~min}\right)\right]$.

La microfiltration tangentielle a été réalisée sur le jus brut en recirculation totale. Les perméats et le rétentat ont été recyclés en continu vers le réservoir d'alimentation. Ainsi le facteur de réduction volumique est
Figure 1.

Schéma de l'installation pilote de microfiltration tangentielle utilisée pour tester quatre membranes minérales vis-à-vis de la clarification du jus de pommes de cajou. 


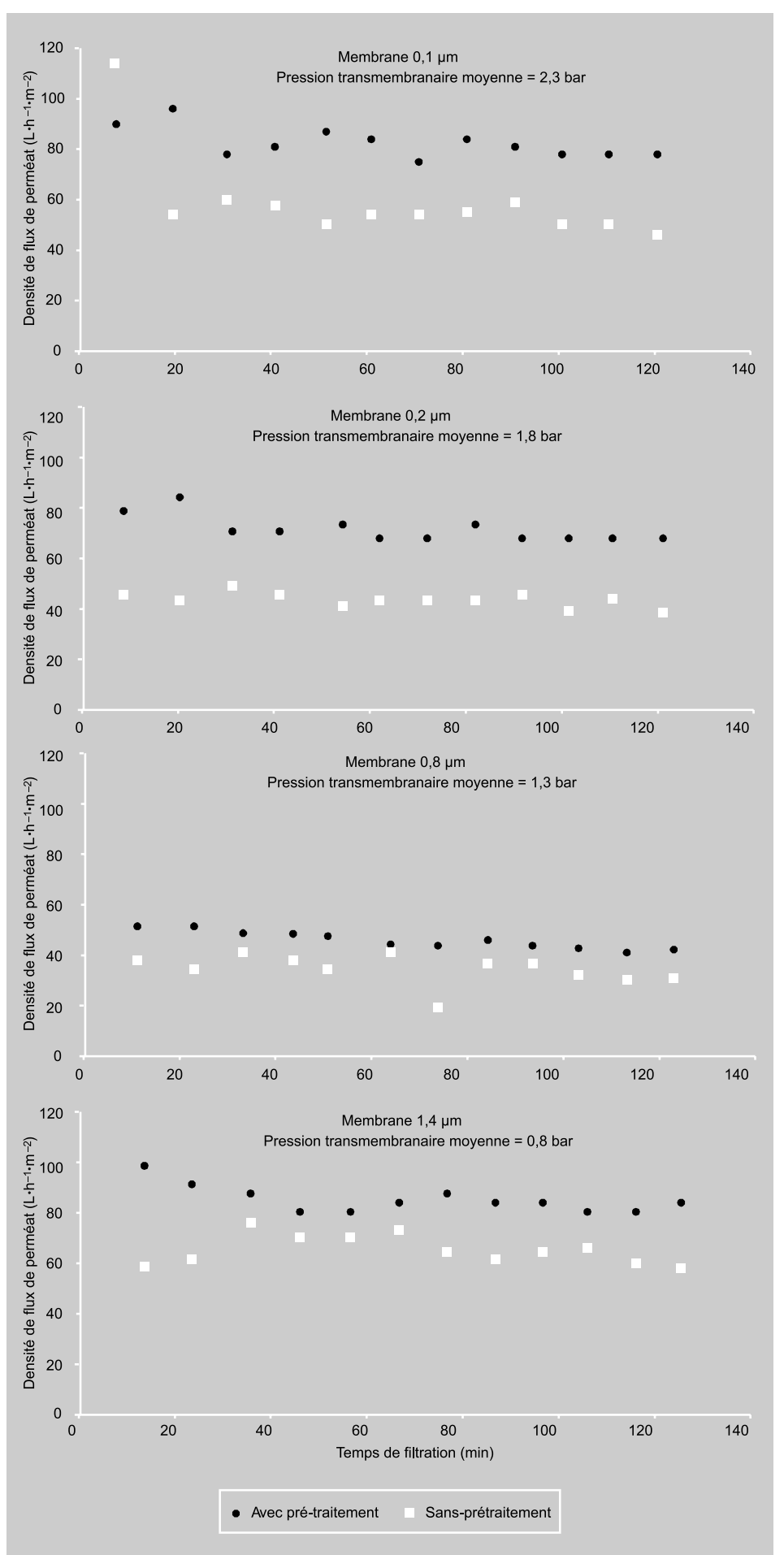

Figure 2.

Évolution de la densité de flux de perméat au cours de la filtration du jus de pommes de cajou avec et sans prétraitement enzymatique sur quatre membranes différenciées par le diamètre de leurs pores. resté voisin de 1 . Les densités de flux de filtrat obtenues sur les quatre membranes ont été mesurées manuellement pendant $2 \mathrm{~h}$. Des prélèvements de $100 \mathrm{~mL}$ de rétentat et de perméats ont été effectués en fin d'essai afin de caractériser les produits obtenus.

Pour évaluer l'intérêt d'une liquéfaction enzymatique partielle avant la filtration, des essais comparatifs avec et sans prétraitement enzymatique ont été réalisés. En se référant aux résultats obtenus par Vaillant et al. [11], une préparation commerciale équilibrée en cellulases et pectinases a été choisie : Rapidase Liq+ (Gist Brocades, France). La liquéfaction a été réalisée pendant 1 h 30 à $35^{\circ} \mathrm{C}$ dans un bain-marie en utilisant une dose d'enzyme de $1 \mathrm{~mL}$ par litre de jus à traiter.

\subsection{Analyses physico-chimiques}

La teneur en acide ascorbique a été déterminée par titration au 2,6-dichlorophénol indophénol en utilisant de l'acide oxalique comme stabilisant. La teneur en tannins condensés a été mesurée en utilisant la méthode par réaction avec la vanilline décrite par Deschamps et Cheryan [13]. Le $\mathrm{pH}$ des jus a été mesuré à l'aide d'un $\mathrm{pH}$ mètre Jenco (modèle 6072) et la turbidité à l'aide d'un turbidimètre Hach (43900 Ratio/ XR). La couleur a été évaluée à l'aide d'un chromamètre Minolta (CR-A70) dans le système $L^{*}, a^{*}, b^{*}$. Le degré Brix du jus brut et des produits clarifiés obtenus a été mesuré à l'aide d'un réfractomètre portable Atago 0-32 ${ }^{\circ}$ Brix.

\section{Résultats}

\subsection{Performances du procédé}

Les évolutions des densités de flux de perméats au cours de la filtration sur les quatre membranes testées ont été comparées (figure 2).

Dans tous les cas, les densités de flux se sont stabilisées après 20 min de filtration. Le colmatage des membranes atteint donc rapidement un équilibre. Pour comparer les résultats, il est ainsi possible de raisonner sur les 


\begin{tabular}{|c|c|c|c|}
\hline \multirow[t]{2}{*}{$\begin{array}{l}\text { Diamètre de pores } \\
(\mu \mathrm{m})\end{array}$} & \multirow[t]{2}{*}{$\begin{array}{l}\text { Pression transmembranaire } \\
\text { (bar) }\end{array}$} & \multicolumn{2}{|c|}{$\begin{array}{l}\text { Densité de flux de perméat } \\
\qquad\left(L \cdot h^{-1} \cdot m^{-2}\right)\end{array}$} \\
\hline & & Sans pré-traitement & Avec pré-traitement \\
\hline 0,1 & 2,3 & 52 & 79 \\
\hline 0,2 & 1,8 & 46 & 75 \\
\hline 0,8 & 1,3 & 36 & 47 \\
\hline 1,4 & 0,8 & 50 & 68 \\
\hline
\end{tabular}

densités de flux moyennes calculées sur la dernière demi-heure de filtration (tableau I). Sans prétraitement enzymatique, les densités de flux atteintes ont été proches de $50 \mathrm{~L} \cdot \mathrm{h}^{-1} \cdot \mathrm{m}^{-2}$ pour toutes les membranes testées sauf pour la membrane $0,8 \mu \mathrm{m}$ pour laquelle elle a été significativement plus faible $\left(36 \mathrm{~L} \cdot \mathrm{h}^{-1} \cdot \mathrm{m}^{-2}\right)$. Dans les conditions testées, l'utilisation de membranes de grand diamètre de pores n'apporte donc pas d'amélioration significative en termes de performances. Il est probable que le colmatage interne des membranes par les particules en suspension contenues dans le jus brut est favorisé lorsque le diamètre moyen des pores est plus élevé. Notons cependant que la pression transmembranaire est un paramètre opératoire qu'il serait nécessaire d'optimiser pour chacune des membranes utilisées.

Le prétraitement enzymatique a amélioré considérablement les densités de flux de perméats pour les quatre membranes. En effet, les densités de flux obtenues ont été augmentées de (50 à 60) \% pour les membranes présentant des pores de $(0,1$ et $0,2) \mu \mathrm{m}$ et de l'ordre de $30 \%$ pour les membranes à pores de $(0,8$ et 1,4$) \mu \mathrm{m}$. Ces résultats sont liés à l'hydrolyse des polyosides pariétaux, constituants majoritaires de la fraction insoluble des jus de fruits [14]. Cette hydrolyse conduit non seulement à une diminution de la viscosité du jus mais également à une diminution de son pouvoir de colmatage. Comme dans le cas de beaucoup de jus de fruits pulpeux, l'intérêt de coupler un traitement enzymatique à la filtration du jus de pomme de cajou est donc nettement mis en évidence. Utilisées sur le produit avec prétraitement enzymatique à des pressions transmembranaires respectives de $(2,3 ; 1,8$ et 0,8$)$ bar, les membranes à pores de $(0,1 ; 0,2$ et 1,4$) \mu \mathrm{m}$ ont conduit à des densités de flux voisines, voire supérieures, à $70 \mathrm{~L} \cdot \mathrm{h}^{-1} \cdot \mathrm{m}^{-2}$. Ces valeurs sont d'autant plus intéressantes dans l'optique d'une application industrielle du procédé qu'elles devraient pouvoir être encore améliorées en optimisant les conditions opératoires, notamment la pression transmembranaire. En revanche, la densité de flux obtenue sur la membrane à pores de $0,8 \mu \mathrm{m}$ et à pression transmembranaire de 1,3 bar est significativement plus faible. Compte tenu de leur diamètre de pores, les membranes à $(0,1$ et 0,2$) \mu \mathrm{m}$ garantissent théoriquement la stérilité du jus clarifié ce qui représente un avantage supplémentaire considérable par rapport aux membranes à pores de $(0,8$ et 1,4$) \mu \mathrm{m}$. Il serait cependant souhaitable de vérifier expérimentalement la stabilisation microbiologique du produit. Enfin, pour mieux évaluer les performances des quatre membranes choisies, l'optimisation de la pression transmembranaire devrait être envisagée pour chacune des membranes (comparaison des densités de flux obtenues aux pressions transmembranaires optimales).

\subsection{Qualité des produits obtenus}

Les principales caractéristiques des jus clarifiés obtenus (perméats) avec prétraitement enzymatique ont été comparées à celles du 
Tableau II.

Principales caractéristiques physico-chimiques du jus brut de pomme de cajou et des divers jus clarifiés obtenus par microfiltration tangentielle avec prétraitement enzymatique $(\mathrm{pH}=4,4$ pour tous les produits).

\begin{tabular}{|c|c|c|c|c|c|c|c|c|}
\hline \multirow[t]{2}{*}{ Echantillon } & \multirow{2}{*}{$\begin{array}{l}\text { Diamètre de pore } \\
\text { de la membrane } \\
\qquad(\mu \mathrm{m})\end{array}$} & \multirow{2}{*}{$\begin{array}{l}{ }^{\circ} \text { Brix } \\
(\%)\end{array}$} & \multirow{2}{*}{$\begin{array}{c}\text { Vitamine C } \\
\left(\mathrm{mg} \cdot 100 \mathrm{~mL}^{-1}\right)\end{array}$} & \multirow{2}{*}{$\begin{array}{l}\text { Tannins condensés } \\
\left(\mathrm{mg} \cdot 100 \mathrm{~mL}^{-1}\right)\end{array}$} & \multirow{2}{*}{$\begin{array}{l}\text { Turbidité } \\
\text { (UNT) }\end{array}$} & \multicolumn{3}{|c|}{ Paramètre de couleur } \\
\hline & & & & & & $L^{*}$ & $a^{*}$ & $b^{*}$ \\
\hline Jus brut & - & $10,7( \pm 0,1)$ & $208( \pm 5)$ & $259( \pm 12)$ & $3120( \pm 184)$ & $35,2( \pm 0,2)$ & $-1,3( \pm 0,1)$ & $-0,9( \pm 0,2)$ \\
\hline \multirow{4}{*}{$\begin{array}{l}\text { Jus } \\
\text { clarifiés }\end{array}$} & 0,1 & 9,8 & 204 & 5 & 0,2 & 27,7 & $-0,2$ & $+0,3$ \\
\hline & 0,2 & 9,8 & 204 & 7 & 0,4 & 27,8 & $-0,2$ & $+0,3$ \\
\hline & 0,8 & 9,6 & 192 & 8 & 0,3 & 27,6 & $-0,2$ & $+0,3$ \\
\hline & 1,4 & 10,0 & 215 & 6 & 0,3 & 27,8 & $-0,2$ & $+0,3$ \\
\hline
\end{tabular}

jus brut (tableau II). Le $\mathrm{pH}$ du jus de fruit n'a pas été modifié par le traitement $(\mathrm{pH}=$ 4,4 pour tous les produits). Les acides organiques ne sont donc pas retenus. L'extrait sec soluble ( $\left.{ }^{\circ} \mathrm{Brix}\right)$ mesuré sur les perméats a été réduit de (6 à 10) \% par rapport au jus brut. Compte tenu des diamètres de pores utilisés, une rétention significative des sucres - solutés majoritaires dans ce type de produit - est peu probable. Les différences observées sont vraisemblablement liées à la méthode analytique utilisée (réfractométrie) car la présence de pulpe dans le produit peut interférer de façon significative sur cette mesure. Les teneurs en acide ascorbique (vitamine C) des perméats ont été très voisines de celle du jus brut. Pour toutes les membranes, les pertes mesurées par rapport à la teneur du jus brut ont été inférieures à $2 \%$ sauf pour la membrane à pores de $0,8 \mu \mathrm{m}$ pour laquelle elles ont été de $8 \%$. Ici encore, les diamètres de pores mis en oeuvre n'ont pas permis d'envisager une rétention significative de ce soluté. Les légères pertes en acide ascorbique détectées ne peuvent donc être expliquées que par une dégradation oxydative liée à la mise en contact du produit avec l'oxygène de l'air.

Les perméats obtenus ont présenté des teneurs en tannins condensés beaucoup plus faibles que celles du jus brut. La rétention de ces composés polyphénoliques, responsables de l'astringence du produit, a été d'environ $98 \%$ pour toutes les membranes testées quel que soit leur diamètre de pores. Malgré des masses molaires plus élevées que les sucres (entre 1 et $18 \mathrm{kDa}$ environ), les seuils de coupures des membranes utilisées ne permettent pas d'expliquer la rétention de ces solutés. L'association des tannins avec des composés insolubles qui, eux, sont retenus par les membranes (notamment les polyosides pariétaux, constituants majoritaires de la pulpe), pourrait en revanche expliquer ces résultats. Cette hypothèse demanderait néanmoins à être vérifiée. La microfiltration tangentielle permet de produire du jus clarifié de pomme de cajou avec des teneurs en tannins inférieures à 0,01\%. Ces résultats sont comparables à ceux obtenus en utilisant un collage à la gélatine suivi d'une filtration frontale, procédé classiquement mis en oeuvre pour réduire l'astringence du produit [3]. Avec une efficacité comparable, la microfiltration tangentielle sur membrane céramique permet donc de s'affranchir de l'étape de collage ce qui présente un intérêt technologique important. La très faible teneur en tannins du jus clarifié obtenu permet de proposer un produit qui n'est plus astringent et qui est également plus stable. En effet, les tannins peuvent être responsables de la formation de troubles lors du stockage du jus de fruit.

Les jus clarifiés (perméats) sont parfaitement limpides comme en attestent les valeurs de turbidité obtenues $(<1$ unité néphélométrique de turbidité). Dans la gamme d'étude, 
le diamètre de pores n'a pas eu d'influence significative sur l'efficacité de la clarification. Pour les quatre membranes testées, le taux de clarification a été supérieur à 99,9\%. Compte tenu de la rétention quasiment totale de la pulpe, la couleur du jus clarifié est évidemment très différente de celle du jus brut. Les jus clarifiés obtenus ont été beaucoup moins colorés et jaune pâle. Leurs valeurs $L^{*}$, $a^{*}, b^{*}$ ont été semblables pour les quatre membranes.

À partir d'une première évaluation sensorielle rapide, l'odeur des perméats s'est révélée très voisine de celle du jus brut. Les jus clarifiés possèdent donc une qualité aromatique assez proche de celle du jus initial. Néanmoins, afin de confirmer ces résultats, des tests sensoriels plus approfondis, associés à une caractérisation de la composition en composés d'arôme des différents produits, doivent être envisagés.

\section{Conclusion}

Notre étude a permis de vérifier les potentialités de la microfiltration tangentielle sur membranes minérales pour la clarification du jus de pomme de cajou. Quel que soit le diamètre des pores de la membrane utilisée, le procédé testé a conduit à l'obtention d'un jus de fruit parfaitement limpide et ne présentant pas de diminution significative de teneur en vitamine $\mathrm{C}$; de plus, la technique a permis d'éliminer la quasi-totalité des composés phénoliques responsables de l'astringence du produit. Les densités de flux, dépendantes des conditions opératoires utilisées, ont été comprises entre (36 et 79) $\mathrm{L} \cdot \mathrm{h}^{-1} \cdot \mathrm{m}^{-2}$. Le couplage de la microfiltration avec un prétraitement enzymatique de liquéfaction a amélioré les performances de la filtration de (30 à 60) \% selon les cas.

Parmi les quatre membranes testées, les membranes présentant un diamètre moyen de pores de $(0,1$ et 0,2$) \mu \mathrm{m}$, utilisées à des pressions transmembranaires de $(2,3$ et 1,8) bar, respectivement, ont conduit aux densités de flux les plus élevées. Les valeurs obtenues, supérieures à $70 \mathrm{~L} \cdot \mathrm{h}^{-1} \cdot \mathrm{m}^{-2}$, sont intéressantes pour envisager une utilisation industrielle. Compte tenu de leur faible dia- mètre de pores, ces deux membranes présentent également l'avantage de stériliser le produit.

Une étude complémentaire devra être envisagée pour l'utilisation du procédé à des fins d'une future application industrielle. L'optimisation du traitement enzymatique et des conditions opératoires de la filtration est indispensable. Cette approche devra considérer simultanément les performances du procédé et les coûts de production. Des essais en concentration doivent également être menés afin de déterminer l'impact du facteur de réduction volumique sur les résultats. Enfin, la caractérisation de la qualité sensorielle du jus de pomme de cajou clarifié obtenu doit être approfondie afin de mieux évaluer l'acceptabilité du produit auprès des clients potentiels.

\section{Références}

[1] Anonymous, Statistical Databases (FAOSTAT), FAO, Roma, Italy, http://apps.fao.org, 2001.

[2] Paiva F.F.A., Garruti D.S., Silva Neto R.M., Aproveitamento industrial do caju, Embrapa Agroindústria Tropical / Sebrae, Fortaleza, Brasil, 2000.

[3] Lautié E., Dornier M., de Souza Filho M., Reynes M., Les produits de l'anacardier : caractéristiques, voies de valorisation et marchés, Fruits 56 (2001) 235-248.

[4] Leite L.A., A agroindustria do caju no Brasil : políticas públicas e transformações econômicas, Embrapa / Cnpat, Fortaleza, Brasil, 1994.

[5] Anonymous, Campanha nacional de aumento da produtividade do cajueiro e produtos derivados do cajueiro, Embrapa / Cnpat, Fortaleza, Brasil, 1992.

[6] Kubo I., Ochi M., Vieira P.C., Komatsu, S., Antitumor agents from the cashew (Anacardium occidentale) apple juice, J. Agric. Food Chem. 41 (1993) 1012-1015.

[7] Muroi H., Kugo A., Kubo I., Antimicrobial activity of cashew apple flavor compounds, J. Agric. Food Chem. 41 (1993) 1106-1109.

[8] Kubo J., Ran Lee J., Kubo I., Anti-Helicobacter pylori agents from the cashew apple, J. Agric. Food Chem. 47 (1999) 533-537. 
[9] Pimentel C.R.M., Castanha de caju : produção e consumo internacional, EMBRAPA / Cnpca, Fortaleza, Brésil, 1992.

[10] Vaillant F., Millan A., Dornier M., Decloux M., Reynes M., Strategy for economical optimisation of the clarification of pulpy fruit juices using crossflow microfiltration, J. Food Engin. 48 (2001) 83-90.

[11] Vaillant F., Millan P., O'Brien G., Dornier M., Decloux M., Reynes M., Crossflow microfiltration of passion fruit juice fruit after partial enzymatic liquefation, J. Food Engin. 42 (1999) 215-224.
[12] Girard B., Fukumoto L., Membrane processing of fruit juices and beverages: a review, Crit. Rev. Food Sci. 40 (2000) 91-157.

[13] Deschamps S.S., Cheryan M., Evaluation of vanillin assay for tannins analysis of dry beans, J. Food Sci. 50 (1985) 905-910.

[14] Vaillant F., Clarification et concentration de jus de fruits tropicaux pulpeux associant traitements enzymatiques, microfiltration tangentielle et évaporation osmotique, Ensia, thèse de doctorat, spécialité Génie des Procédés, Massy, France, 2000, 247 p.

\section{Aplicación de la microfiltración tangencial a la producción de jugo clarificado de marañón por medio de membranas minerales.}

Resumen - Introducción. El marañón es una materia prima poco utilizada ya que se le ha considerado por lo general como un sub-producto del procesamiento de la semilla de marañón. Entre las distintas alternativas tecnológicas posibles para la clarificación de jugos de frutas pulposos, la microfiltración tangencial mediante el uso de membranas minerales es un proceso prometedor. El objetivo de nuestro trabajo fue evaluar el interés de este proceso para la producción de jugo de marañón clarificado. Materiales y métodos. Los ensayos de filtración se realizaron a $30{ }^{\circ} \mathrm{C}$ empleando un equipo piloto a escala de laboratorio provisto de distintas membranas tubulares de alúmina. Se evaluaron cuatro membranas de diferente diámetro promedio de poro $[(0.1 ; 0.2 ; 0.8$ y 1.4$) \mu \mathrm{m}]$. Se compararon los rendimientos (densidad de flujo de permeado) y la composición del jugo clarificado obtenido (taninos condensados, acidez total, ácido ascórbico, pH, turbidez, color y extracto seco soluble) para cada una de las membranas. Se evaluó también el uso de un pretratamiento enzimático de licuefacción acoplado a la microfiltración. Resultados y discusión. Las densidades de flujo de permeado obtenidas oscilaron en un rango comprendido entre $\left[(36\right.$ y 79$\left.) \mathrm{L} \cdot \mathrm{h}^{-1} \cdot \mathrm{m}^{-2}\right]$. Los mejores resultados se obtuvieron con las membranas de $(0.1$ y 0.2$) \mu \mathrm{m}$. El acoplamiento de la microfiltración a un pretratamiento enzimático de licuefacción aumentó los rendimientos de la filtración de un $(30$ a un 60$) \%$. Todas las membranas permitieron la obtención de un jugo completamente clarificado cuyo contenido de ácido ascórbico fue similar al del jugo fresco. Por otra parte, los compuestos fenólicos, presentes en gran cantidad en el jugo de marañón empleado como materia prima, fueron prácticamente eliminados durante el proceso, lo que provocaría una disminución considerable de la astringencia del jugo. Conclusión. Nuestro trabajo permitió verificar el potencial del proceso de microfiltración tangencial para la producción de jugo clarificado de marañón. Sin embargo, antes de considerar una futura aplicación industrial, es necesario optimizar las condiciones de tratamiento y caracterizar mejor tanto la calidad nutricional como sensorial del jugo de marañón clarificado.

Brasil / Anacardium occidental / marañón / jugo de frutas / clarificación / microfiltración / membranas / polifenoles 